\title{
Combining Cooperative Relaying with Spectrum Sensing in Cognitive Radio Networks
}

\author{
Tuan Do and Brian L. Mark* \\ Dept. of Electrical and Computer Engineering \\ George Mason University, MS 1G5 4400 University Drive, Fairfax, VA \\ *Correspondent: tel 703-993-4069; fax 703-993-1601; email bmark@ gmu.edu
}

\begin{abstract}
In a network with dynamic spectrum access, secondary users equipped with frequency-agile cognitive radios communicate with one another via spectrum that is not being used by the primary, licensed users of the spectrum. We consider a scenario in which a secondary transmitter can communicate with a secondary receiver via a direct communication link or a relay channel, depending on the state of a primary transmitter. We develop a cooperative communications strategy that exploits the presence of spectrum holes both in time and in space by combining cooperative relaying with joint spatial-temporal spectrum sensing. A strategy based on pure temporal sensing alone uses the direct link when the primary transmitter is off, whereas a scheme based on spatial sensing alone uses the relay channel. Our numerical results show that the proposed scheme significantly reduces the average symbol error probability compared to schemes based on pure temporal or spatial sensing.

Index Terms-Cognitive radio, cooperative communications, dynamic spectrum access
\end{abstract}

\section{INTRODUCTION}

Recent empirical studies of radio spectrum usage have shown that licensed spectrum is typically highly underutilized [1]. Cognitive radio, first introduced in [2] is a promising technology for increasing spectral efficiency in wireless systems. In this paper, we consider cognitive radios that have the ability to identify portions of unused spectrum and dynamically tune to frequency channels within such spectrum holes. Such radios are said to be frequency-agile or spectrum-agile.

Spectrum holes exist both in time and in space. A temporal spectrum hole may arise, for example, when a licensed or primary user of the spectrum is idle, i.e., not transmitting. In this case, temporal spectrum hole is the duration for which the primary transmitter is in the idle or OFF state. A spatial spectrum hole with respect to a given frequency channel may occur if a given secondary user is sufficiently far from a primary user that is actively transmitting. In this case, the secondary user may transmit up to a certain level, which we called the maximum interference-free transmit power (MIFTP), without causing harmful interference to primary users who are receiving the transmissions. In an earlier paper [3], a joint spatialtemporal sensing was proposed. In the proposed scheme, a secondary node performs spatial sensing to determine its MIFTP when the primary transmitter is ON and uses localization information obtained in the process of spatial sensing to improve the performance of temporal sensing, which estimates the ON/OFF state of the primary transmitter.

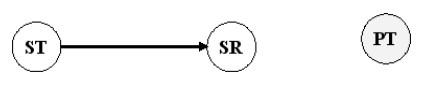

(a) Direct communication.

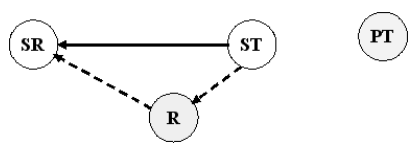

(b) Single relay cooperative communication.

Fig. 1. Joint spatial-temporal sensing via cooperative communication.

In this paper ${ }^{1}$, we propose a cooperative communication strategy that employs joint spatial-temporal sensing to maximize the transmission capacity of secondary users in a cognitive radio network. In Fig. 1(a), the secondary transmitter (ST) communicates directly with the secondary receiver (SR), due to the existence of a spatial spectrum hole with respect to the primary transmitter (PT). However, in the scenario depicted in Figs. 1(b), ST communicates directly with SR only when PT is in the OFF state. In these scenarios, when PT is ON, ST transmits to SR via a relay channel. By enabling the use of both the direct and relay channels, the transmission performance of the secondary system can be improved significantly.

Cooperative relay communications or cooperative diversity has received a lot of attention in recent years (cf. [4][6]). Two well-known cooperative strategies are amplifyand-forward (AF) and decode-and-forward (DF). In this paper, we consider only the AF strategy, which achieves

\footnotetext{
${ }^{1}$ This work was supported in part by the U.S. National Science Foundation under Grants CNS-0520151 and ECS-0246925.
} 


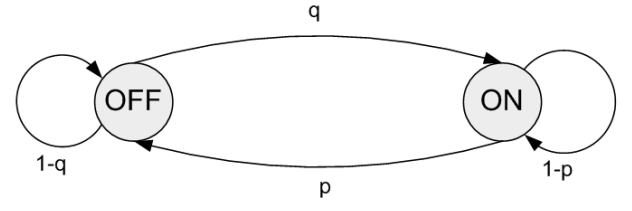

Fig. 2. 2-state Markov chain model for PT ON/OFF process.

diversity by using maximal ratio combining. Furthermore, we focus on the case of a single-hop relay channel.

The remainder of the paper is organized as follows. Section II describes the system model. Section III discusses the performance of the system. Section IV presents simulation results. Finally, the paper is concluded in Section V.

\section{SySTEM MOdEL}

\section{A. Transmission frames and PT behavior}

Time on the wireless channel is divided into frames consisting of $N_{s}$ symbols. We shall assume perfect symbollevel timing synchronization between the nodes of the secondary system. The case of imperfect synchronization has been studied in [6]. The PT alternates between the ON and OFF states on a per-frame basis according to the on-off Markov model of Fig. 2.

The ON and OFF durations can be modeled by geometric random variables with parameters $q$ and $p$, respectively (cf. [7]). We focus on scenarios in which $q>p$; i.e., on average, PT is more often in the ON state than the OFF state. Let us suppose that $q=K p$, where $K$ is an integer with $K>1$. Hence, if PT is OFF for one time frame on average, it will be $\mathrm{ON}$ for $\mathrm{K}$ time frames, on average. A similar approach can be used when $p>q$.

\section{B. Cooperative transmission protocol}

Suppose ST desires to transmit $N_{s}$ symbols; i.e., it requires one full frame in which PT is OFF. The cooperative protocol works as follows (cf. Fig. 1(b)): When PT is ON, the relay nodes transmit information to SR. When PT is OFF, ST sends information directly to SR. In order to achieve this, the secondary nodes perform joint spatialtemporal sensing, as discussed in [3]. In particular, all secondary users estimate their MIFTPs based on signal strength measurements, which they exchange with one another. They also decide whether the PT is ON or OFF, by transmitting their local decisions to a fusion center, which then makes the final decision.

When PT is ON, ST cannot communicate directly with SR. We shall assume that there exists a communications path from ST to SR through a relay node R. This path would be established by a routing protocol. A repetition code (cf. [8]) is used to repeat the transmission of the signal over $K_{0}$ and $K_{1}$ frames, where $K_{0}+K_{1}=K$. At each relay, the transmitted signal is amplified and forwarded to the next relay node until it reaches the destination.

When PT is OFF, ST can communicate $N_{s}$ symbols over one time frame directly to SR. Due to broadcast wireless channel, $\mathrm{R}$ also received a copy of signal from ST but ignores it. When SR receives a signal from the relay path, it stores the received signal and waits until it receives the same signal directly from ST and vice versa. The received signals at $\mathrm{SR}$ are then combined using maximal ratio combining (MRC). Finally, maximum-likelihood detection is used to detect the signals.

\section{Cooperative relaying}

The received signal of a simple wireless channel model with flat fading and no shadowing is given by [9]

$$
y=\delta \sqrt{\left(\frac{d_{r}}{d}\right)^{\alpha}} h \sqrt{\epsilon} s+n=\sqrt{P} h s+n,
$$

where $\delta^{2}$ is the free space signal-power attenuation factor between the source and a reference distance $d_{r}, d$ is the distance between the source and destination, $\alpha$ is the propagation exponent, $h \sim \mathcal{C N}\left(0, \sigma_{h}^{2}\right)$ is a complex Gaussian random variable with variance $\sigma_{h}^{2}, n \sim \mathcal{C N}\left(0, N_{0}\right)$, and $s$ is the transmitted signal. In (1), $P=\delta^{2}\left(\frac{d_{r}}{d}\right)^{\alpha} \epsilon$ denotes the equivalent transmitted power after taking into account the effect of path loss. We also define

$$
P_{0}=\delta^{2}\left(\frac{d_{r}}{d_{0}}\right)^{\alpha} \epsilon_{0}, P_{1}=\delta^{2}\left(\frac{d_{r}}{d_{1}}\right)^{\alpha} \epsilon_{1}, P_{t}=\delta^{2}\left(\frac{d_{r}}{d_{t}}\right)^{\alpha} \epsilon_{t},
$$

as the equivalent transmitted powers from ST to R, from $\mathrm{R}$ to SR, and from ST to SR, respectively. Here, $d_{0}, d_{1}$ and $d_{t}$ denote the distances between the node pairs (ST,R), (R,SR), and (ST,SR), respectively. Also, $\epsilon_{0}$ and $\epsilon_{1}$ denote the MIFTPs of $S T$ and $R$, respectively, when PT is ON and $\epsilon_{t}$ denotes the maximum transmit power of ST. When $\epsilon_{0} \ll \epsilon_{t}$, ST may not communicate directly with SR when $\mathrm{PT}$ is $\mathrm{ON}$ because the power level $P_{0}$ is too low. Hence, when PT is ON, ST communicates with SR through relay node $\mathrm{R}$, which is closer to ST.

The received signal at a relay is the MRC sum of a repetition code over $K_{0}$ time frames [8]:

$$
y_{1}=\sum_{i=1}^{K_{0}} g_{i}^{*}\left(g_{i} \sqrt{P_{0}} s+n_{i}\right)=\tilde{g} \sqrt{P_{0}} s+\tilde{n},
$$

where $\tilde{g}=\sum_{i=1}^{K_{0}}\left|g_{i}\right|^{2}$ and $\tilde{n}=\sum_{i=1}^{K_{0}} g_{i}^{*} n_{i}, s$ is the transmitted symbol, $|s|^{2}=1$ and $g_{i}$ is the channel gain between $\mathrm{ST}$ and $\mathrm{R}$ during time frame $i$. The received signal at $\mathrm{SR}$ due to relay $\mathrm{R}$ is

$$
\begin{aligned}
y_{R} & =\sum_{j=1}^{K_{1}} h_{j}^{*}\left(\sqrt{P_{1}} h_{j} A\left(\tilde{g} \sqrt{P_{0}} s+\tilde{n}\right)+n_{j}\right) \\
& =\sqrt{P_{0} P_{1}} \tilde{h} A \tilde{g} s+n_{R},
\end{aligned}
$$


where $\tilde{h}=\sum_{j=1}^{K_{1}}\left|h_{j}\right|^{2}, n_{R}=\sum_{j=1}^{K_{1}}\left(\left|h_{j}\right|^{2} A \sqrt{P_{1}} \tilde{n}+\right.$ $\left.h_{j}^{*} n_{j}\right), h_{j}$ is the channel gain between R and SR during time frame $j$. Here, $A$ is the amplification factor which is chosen to maintain average constant power output at $\mathrm{R}, A^{2}=1 /\left(P_{0} \tilde{g}^{2}+N_{0} \tilde{g}\right)$. The noise variance of $y_{R}$, $\sigma_{R}^{2}=A^{2} P_{1} \tilde{g} \tilde{h}^{2} N_{0}+\tilde{h} N_{0}$ where $\tilde{h}=\sum_{j=1}^{K_{1}}\left|h_{j}\right|^{2}$.

The direct transmission (ST $\rightarrow \mathrm{SR}$ ) channel model is

$$
y_{S T}=f \sqrt{P_{t}} s+n_{S T} .
$$

where $f$ is the channel gain between ST and SR. $f$, $h_{j}$ and $g_{i}$ are constant over one time frame duration and independently identical distributed from one frame to another. At SR, MRC is used to combine $y_{R}$ and $y_{S T}$. The noise variables $n_{R}$ and $n_{S T}$ have different powers because $n_{R}$ includes a noise contribution at the relay. For this reason, noise normalization is necessary for MRC of $y_{S T}$ and $y_{R}$ [5]. The resulting SNR is

$$
\gamma_{w}=|f|^{2} \frac{P_{t}}{N_{0}}+|A \tilde{g} \tilde{h}|^{2} \frac{P_{0} P_{1}}{\sigma_{R}^{2}}=\gamma_{t}+\gamma_{r},
$$

where $\gamma_{t}=|f|^{2} \frac{P_{t}}{N_{0}}$ and

$$
\gamma_{r}=|A \tilde{g} \tilde{h}|^{2} \frac{P_{0} P_{1}}{\sigma_{R}^{2}}=\frac{\gamma_{0} \gamma_{1}}{\gamma_{0}+\gamma_{1}+1},
$$

with $\gamma_{0}=\tilde{g} \frac{P_{0}}{N_{0}}$ and $\gamma_{1}=\tilde{h} \frac{P_{1}}{N_{0}}$. We assume that $f, g_{i}, h_{j}$ are known at receiving end. The symbol error probability (SEP) conditioned on the instantaneous SNR $\gamma_{w}$ is given by $P_{e}=Q\left(\sqrt{k \gamma_{w}}\right)$ [5] where $k$ is a constant that depends on the type of modulation and $Q(x)=$ $(1 / \sqrt{2 \pi}) \int_{x}^{\infty} e^{-t^{2} / 2} \mathrm{~d} t$ is the standard Q-function.

\section{PERformance ANAlysis}

In this section, we derive a lower bound on the average SEP. We show that the SEP lower bound is minimized when $K_{0}=K_{1}$ for $K$ even and $K_{0}=K_{1}+1$ for $K$ odd.

From (6), we can upper-bound $\gamma_{r}$ as follows:

$$
\gamma_{r} \leq \frac{\gamma_{0} \gamma_{1}}{\gamma_{0}+\gamma_{1}} \leq \frac{\sqrt{\gamma_{0} \gamma_{1}}}{2}=\frac{\sqrt{P_{0} P_{1}}}{2 N_{0}} \sqrt{\tilde{g} \tilde{h}}
$$

Taking expectations on both sides, we have

$$
E\left[\gamma_{r}\right] \leq \frac{\sqrt{P_{0} P_{1}}}{2 N_{0}} E[\sqrt{\tilde{g} \tilde{h}}]
$$

Note that $g_{i}, h_{j} \sim \mathcal{C N}(0,1)$ then $2 \tilde{g}$ and $2 \tilde{h}$ are independent $\chi^{2}$-distributed random variables with $2 K_{0}$ and $2 K_{1}$ degrees of freedom, respectively. Applying Jensen's inequality,

$$
E[\sqrt{\tilde{g} \tilde{h}}] \leq \sqrt{E[\tilde{g} \tilde{h}]}=\frac{1}{2} \sqrt{E[2 \tilde{g}] E[2 \tilde{h}]}=\sqrt{K_{0} K_{1}}
$$

From (8) and (9), we have

$$
E\left[\gamma_{r}\right] \leq \frac{\sqrt{P_{0} P_{1}}}{2 N_{0}} \sqrt{K_{0} K_{1}}
$$

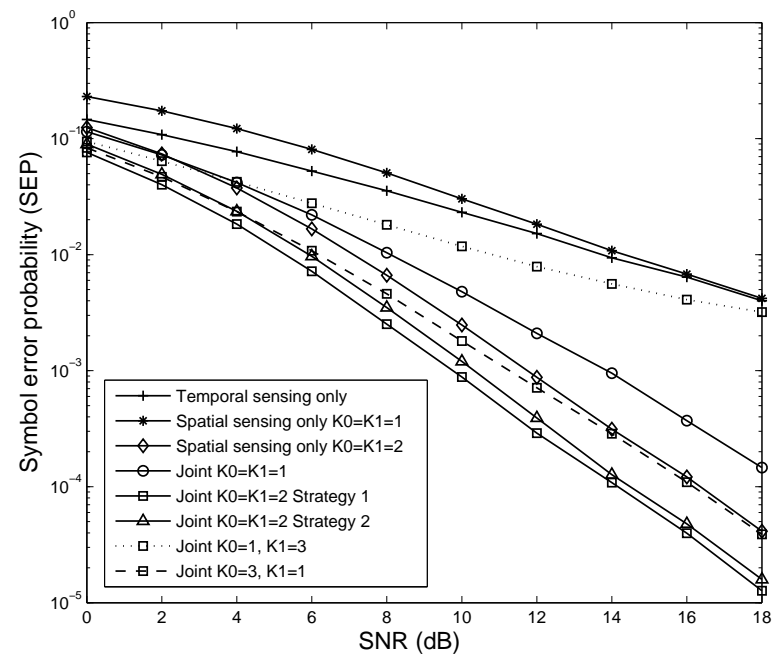

Fig. 3. Amplify-and-Forward (AF) with one relay and $K=4$.

Assuming M-PSK modulation, the average SEP can be expressed as follows [10]:

$$
\mathrm{SEP}=\frac{1}{\pi} \int_{0}^{\frac{(M-1) \pi}{M}} M_{\gamma_{t}}(-\kappa) M_{\gamma_{r}}(-\kappa) \mathrm{d} \theta,
$$

where $M_{\gamma_{t}}(u) \triangleq E\left[e^{u \gamma_{t}}\right]$ and $M_{\gamma_{r}}(u) \triangleq E\left[e^{u \gamma_{r}}\right]$ are the moment generating functions of $\gamma_{t}$ and $\gamma_{r}$, respectively, and $\kappa \triangleq \frac{k}{\sin ^{2} \theta} \geq 0$. Applying Jensen's inequality and (10), we have

$$
M_{\gamma_{r}}(-\kappa)=E\left[e^{-\kappa \gamma_{r}}\right] \geq e^{-\kappa E\left[\gamma_{r}\right]} \geq e^{-\beta \sqrt{K_{0} K_{1}}},
$$

where $\beta \triangleq \kappa \sqrt{P_{0} P_{1}} /\left(2 N_{0}\right) \geq 0$. The lower bound for the SEP is then obtained by substituting the right hand side of (12) into (11).

If $K$ is even, i.e., $K=2 m$ where $m$ is an integer, $2 \sqrt{K_{0} K_{1}} \leq K_{0}+K_{1}=2 m$, with equality holding when $K_{0}=K_{1}=m$. In this case, (12) implies that the choice of $K_{0}=K_{1}$ maximizes the performance of our proposed scheme. If $K$ is odd, i.e., $K=2 m+1$, information-theoretic results in [11] suggest the choice $K_{0}>K_{1}$, in order to maximize the SNR at the first hop. Let $K_{0}=K_{1}+n$, where $n \geq 1$ is an integer. We have $2 K_{1}=2 m+1-n$,

$$
4 K_{0} K_{1}=4 m^{2}+4 m+1-n^{2} \leq 4 m^{2}+4 m .
$$

The equality in (13) holds for $K_{0}=K_{1}+1$, which also suggests that choosing $K_{0}=K_{1}+1$ minimizes $M_{\gamma_{r}}(-\kappa)$ and hence the SEP. Our analysis is confirmed by the simulation results presented in Section IV.

\section{Numerical Results}

In this section, we investigate the performance of the proposed cooperative communication scheme in terms of 


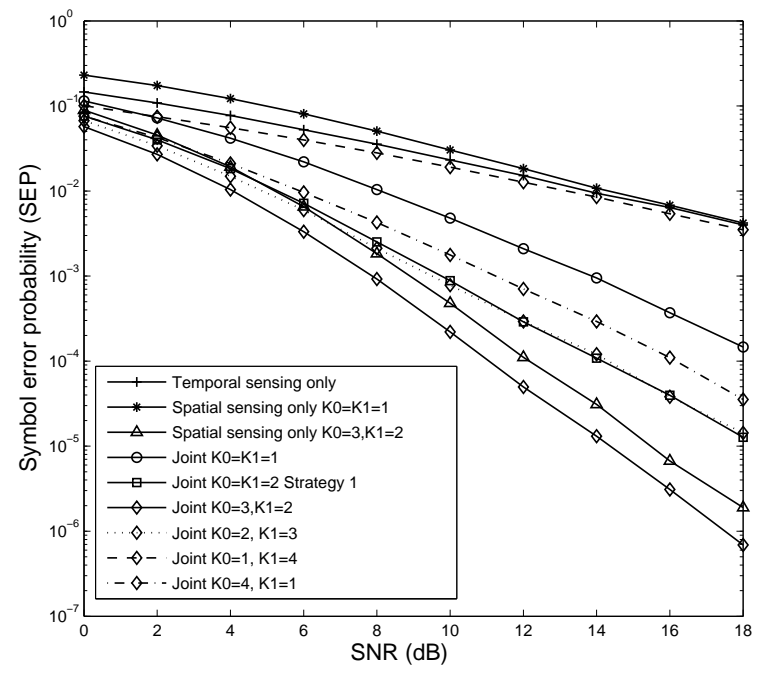

Fig. 4. AF with one relay and $K=5$.

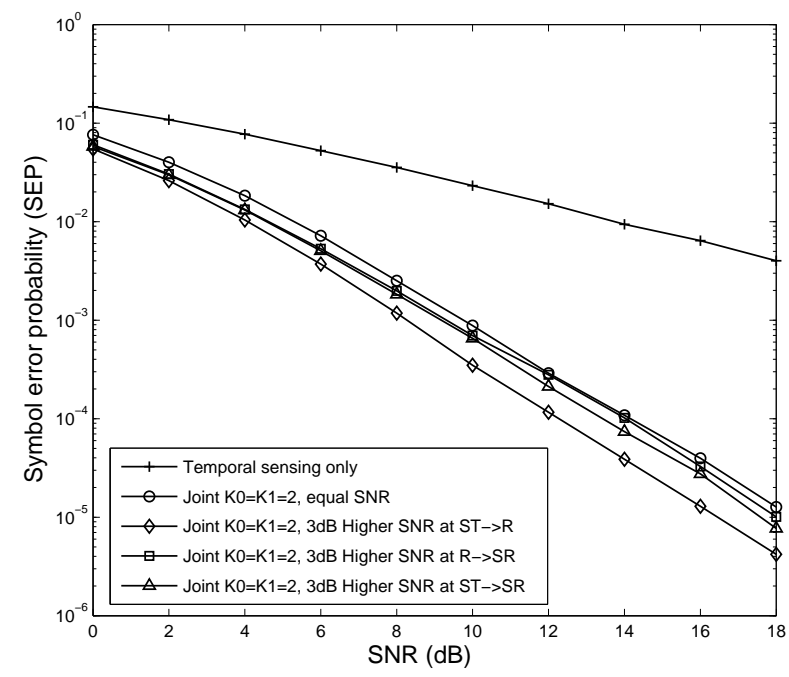

Fig. 5. AF with one relay, asymmetric SNR, and $K=4$.

the average SEP. The results shown in Figs. 3-5 were obtained using a computer simulation in MATLAB. In the performance curves, the $95 \%$ confidence intervals are omitted for clarity. We assume that BPSK modulation is used and $f, g_{i}, h_{j} \sim \mathcal{C N}(0,1)$. The frame length is 100 symbols. Maximal ratio combining and maximum likelihood detection are used at the receiver. Equal SNRs, i.e., $\frac{P_{t}}{N_{0}}=\frac{P_{0}}{N_{0}}=\frac{P_{1}}{N_{0}}$, are assumed in the results presented in Figs. 3 and 4. When $K=2 m$, the transmission strategy follows the scheme described in Section II, which we call (strategy 1). The traditional cooperative communication scheme, which we call strategy 2, transmits $m$ times on the path ST $\rightarrow \mathrm{R} \rightarrow \mathrm{SR}$ over $m$ branches.
Fig. 3 shows the SEP performance of our scheme with one relay and $K=4$ in comparison to pure temporal sensing, pure spatial sensing, and traditional cooperative communications with $K_{0}=K_{1}=1$. The pure temporal sensing scheme corresponds to direct communication from ST to SR, whereas the pure spatial sensing scheme corresponds to communication over the relay path. The simulation results confirm our analytical conclusion that the best performance is achieved with our proposed scheme with $K_{0}=K_{1}=2$. Similar performance trends can be seen in Fig. 4, for which $K=5$, and $K_{0}=3, K_{1}=2$.

The results shown in Fig. 5 pertain to a scenario in which the average SNRs $\frac{P_{t}}{N_{0}}, \frac{P_{0}}{N_{0}}$, and $\frac{P_{1}}{N_{0}}$, are different. This scenario arises in practice because the MIFTP and the distances between ST and R and R and SR may vary, leading to different average SNRs. In this case, a higher $\mathrm{SNR}$ at link ST $\rightarrow \mathrm{R}$ results in the best performance. This suggests that we should choose the relay node that maximizes the average SNR $\frac{P_{0}}{N_{0}}$ from the subset of relay nodes having the same total average SNR $\frac{P_{0}+P_{1}}{N_{0}}$. This also confirms the results in [11] in which the capacity is achieved when the relay is near the source terminal.

\section{CONCLUSION}

We proposed a cooperative transmission strategy that exploits spatial and temporal spectrum holes in a cognitive radio network. The proposed scheme outperforms transmission schemes based on pure temporal sensing and pure spatial sensing.

\section{REFERENCES}

[1] M. McHenry, "Frequency agile spectrum access technologies," in FCC Workshop on Cognitive Radio, May 2003.

[2] J. Mitola, "Cognitive radio: An integrated agent architecture for software defined radio," Ph.D. dissertation, KTH, Stockholm, Sweden, Dec. 2000.

[3] T. Do and B. L. Mark, "Joint spatial-temporal spectrum sensing for cognitive radio networks," in Proc, Conf. on Information Sciences and Systems (CISS'09), Baltimore, MD, Mar. 2009.

[4] J. N. Laneman and G. W. Wornell, "Distributed space-time-coded protocols for exploiting cooperative diversity in wireless networks," IEEE Trans. Inf. Theory, vol. 49, no. 10, pp. 2415-2425, Oct. 2003.

[5] A. Ribeiro, X. Cai, and G. B. Giannakis, "Symbol error probabilities for general cooperative links," IEEE Trans. Wireless Commun., vol. 4, no. 3, pp. 1246-1273, May 2005.

[6] S. Wei, D. Goeckel, and M. Valenti, "Asynchronous cooperative diversity," IEEE Trans. Wireless Commun., vol. 6, no. 6, pp. 1547 1557, 2006.

[7] A. Motamedi and A. Bahai, "MAC protocol design for spectrumagile wireless networks: Stochastic control approach," in Proc. IEEE DySPAN'07, April 2007, pp. 448-451.

[8] D. Tse and P. Viswanath, Fundamentals of Wireless Communication. Cambridge University Press, 2005.

[9] J. Boyer, D. D. Falconer, and H. Yanikomeroglu, "Multihop diversity in wireless relaying channels," IEEE Trans. Commun., vol. 52, no. 10 , pp. $1820-1830$, Oct. 2004.

[10] M. K. Simon and M.-S. Alouini, Digital Communication over Fading Channels, 2nd ed. Wiley Interscience, 2004.

[11] G. Kramer, M. Gastpar, and P. Gupta, "Capacity theorems for wireless relay channels," in 41 st Allerton Conf. on Commun. Control and Comp., Oct 2003. 